\title{
Jessika Schneider/Stephan Grohs/Christoph Knill Wer hat, dem wird gegeben? Die Implementation des Konjunkturprogramms auf kommunaler Ebene
}

\begin{abstract}
Zusammenfassung
Der Beitrag untersucht die Implementation des deutschen Konjunkturprogramms auf kommunaler Ebene. Die Maßnahmen des Konjunkturpakets II stellen einen hervorragenden Fall zur Untersuchung der intendierten und nichtintendierten Wirkungen zeitlich begrenzter distributiver Policies dar. Am Beispiel der baden-württembergischen Kommunen werden anhand quantitativer Analysen zwei typische nichtintendierte Effekte untersucht: D ie relative U ngleichverteilung der Investitionsmittel unter den Kommunen sowie das Auftreten von Mitnahmeeffekten. Die Analyse zeigt verschiedene Einflussfaktor en auf, die diese Effekte beeinflussen. So variieren Investitionsmittel und Mitnahmeeffekte deutlich nach administrative $r$ Kapazität, Haushaltslage und Gemeindegröße. Die aufgezeigten Zielverschiebungen führen zur abschließenden Frage, ob diese von den Gestaltern des Programms billigend in Kauf genommen oder gar im Sinne einer schnellen Programmdurchführung befördert wurden.
\end{abstract}

Schlagworte: Implementationsforschung, Konjunkturprogramme, Wirtschafts- und Finanzkrise, Kommunalverwaltung, Policy-Forschung

\begin{abstract}
Whoever has will be given more? The Implementation of Economic Stimulus Packages in German Local Governments

The article examines the implementation of the German economic stimulus packages at the local level. The measures of the second economic stimulus package (, Konjunkturpaket II“) provide an excellent test case for the intended and unintended effects of temporary distributive policies. Using the example of B aden-Württemberg municipalities, two typical unintended effects are examined by the use of quantitative methods: The unequal distribution of investment resources among local authorities and the occurrence of deadweight. The analysis identifies various factors that influence these effects. Investment resources and deadweight effects vary significantly according to administrative capacity, financial situation and community size. The identified target displacements lead to the final question whether these were considered acceptable to the designers of the program or were even promoted in terms of a fast implementation.
\end{abstract}

Key words: implementation, economic stimulus packages, economic and fiscal crisis, local government, public policy

\section{Einleitung}

Die Finanz- und K onjunkturkrise der Jahre 2008 und 2009 brachte eine Reihe von neuen Politikinstrumenten hervor, deren Einsatz neu und in ihren Wirkungen unsicher war. Neben der Stabilisierung des Bankensektors und einer Re-Regulierung der Finanzmärkte be- 
inhaltete das neue Set von Steuerungsinstrumenten auch eine Reihe von konjunkturpolitischen Maßnahm en (vgl. Hassel/Lütz 2010; Roos 2009). D ie „, Rückkehr des Staates“ (Heinze 2009) w ar so auch eine Rückkehr zum längst abgelegten Repertoire keynesianisch inspirierter K onjunkturprogramme zur kurzfristigen $\mathrm{N}$ achfragestimulierung (vgl. Gaul 2008). Ein wesentlicher Baustein des so genannten Konjunkturpakets II, das Anfang 2009 verabschiedet wurde, war das Kom munale Investitionsprogramm, das zukunftsorientierte Investitionen des öffentlichen Sektors anstoßen sollte. Dieses Programm kann als Beispiel einer zeitlich begrenzten distributiven Policy betrachtet werden, die für die Im plementationsforschung einen hervorragenden Testfall $\mathrm{zu}$ intendierten und nicht intendierten Wirkungen solcher Maßnahm en bietet. D abei stehen hier nicht die konjunkturellen A uswirkungen der K onjunkturprogramme (vgl. hierzu Barabas u.a. 2009; 2011; Brenke u.a. 2009; Horn u.a. 2009), deren wachstums- und nachhaltigkeitspolitischen Folgen (vgl. Pavel/Proske 2009; Pavel 2009; Schmidt et al. 2009) oder deren fiskalpolitischen Folgen (vgl. Fuest 2009; OECD 2010) im Mittelpunkt. Vielmehr sollen im Weiteren am Beispiel Baden-Württem bergs di e spezifischen Steuerungsw irkungen des Programmdesigns im deutschen Mehrebenensystem untersucht werden.

Dabei soll im Besonderen den Fragen nachgegangen werden, ob

- gemäß der distributiven Intention eine weitgehende Gleichverteilung der Mittel erreicht wurde,

- die Mittel tatsächlich zusätzlich verausgabt wurden oder letztendlich Mitnahmeeffekte zu beobachten waren, und

- wie zu beobachtende Varianz im Mittelabruf und wahrscheinliche Mitnahmeeffekte zu erklären sind.

Das komplexe Zusammenspiel zwischen Bund, Ländern und Kommunen legt zunächst die Vermutung nahe, dass $m$ it einem distributiven Program $m$ zwar ein relativ konfliktfrei und rasch zu implementierendes Programm verabschiedet wurde, dieses aber gleichzeitig Zielverschiebungen und Mitnahm eeffekte bei der implementierenden Ebene, den Kommunen mit sich bringt. Die distributive Ausgestaltung des Konjunkturpakets war eine ty pische „Simplifizierungsstrategie“ (Klenk/Nullmeier 2010, S. 281-285): Die Dämpfung von Verteilungskonflikten erleichterte einerseits di e schnelle Durchführung und Um setzung des mehrere adm inistrative Ebenen übergreife nden Program ms, die von wirtschaftswissenschaftlichen Beobachtern einhellig gefordert wurden (vgl. Elmendorf/Furman 2008; Roos 2009). Aus zahlreichen Im plementationsstudien ist andererseits bekannt, dass Programme, die an Kom munen gerichtet sind, häufig von diesen unterlaufen werden und den lokalen Bedürfnissen angepasst werden (vgl. Wollmann 1983; Grohs 2010). Dies wurde im vorliegenden Fall durch eine sehr flexible Interpretation des Vergaberechts und des Zusätzlichkeitskriteriums erleichtert (vgl. Klenk/Nullmeier 2010, S. 285). Dies legt nahe, dass Kom munen unterschiedlich auf das Anreizprogramm reagieren und es letztendlich zu einer asymmetrischen Verteilung zu Gunsten derjenigen Kom munen kommt, die auf der Klaviatur von Antragsverfahren am versiertesten spielen können und hierzu über die erforderlichen administrativen Kapazitäten verfügen. Letztendlich läuft dies also auf das Matthäus-Prinzip hinaus: „Denn wer da hat, dem wird gegeben werden“ (Matth. 25, 29).

$\mathrm{Ob}$ und unter welchen Bedingungen dieses Phänom en empirisch nachweisbar ist, ist Gegenstand des vorliegenden Beitrags. D abei w ird ein innovativer Weg zur Erfassung 
von Mitnahmeeffekten beschritten, welcher auf einer quantitativen Analy se kommunaler Investitionen am Beispiel der baden-württembergischen Kommunen basiert. Für diese liegen gegenwärtig die zuverlässigsten $\mathrm{D}$ aten vor. Zw ar bescheinigt der Rechnungshof Baden-Württemberg dem Inves titionsprogramm eine fast durchgängige Erfüllung des $\mathrm{Zu}$ sätzlichkeitskriteriums (vgl. Rechnungshof 2011). In Einzelfallstudien w urde jedoch bereits festgestellt, dass es sich z.B. im Falle Bremens bei den durchgeführten Investitionsvorhaben „ganz überwiegend nicht um zusätzliche, sondern lediglich um vorgezogene Vorhaben" (Klenk/Nullmeier 2010, S. 285) gehandelt habe und die vereinnahmten Mittel durch haushaltsplanerische Tricks im Gesamthaushalt so umgelegt wurden, dass das Konjunkturprogramm „wie eine generelle Erhöhung des H aushaltes“ (Klenk/Nullmeier 2010, S. 285) wirkte. Die vorliegende Untersuchung ergänzt diese Einzelfallperspektive auf einer breiten empirischen Basis und versucht so über die bislang nur anekdotisch verfügbare Evidenz hinauszugehen. Dabei steht insbesondere die V arianz des U mgangs der Programmadressaten mit dem Angebot zusätzliche r Investitionsmittel im Fokus, welche auf der Basis klassischer Variablen der Policy- und Implementationsforschung erklärt werden soll.

Hierzu führen wir zunächst kurz in das Programmdesign des Kommunalen Investitionsprogramms ein (2.) und entwickeln unter Rückgriff auf Konzepte der Policy - und Im plementationsforschung (3.) Hypothesen zum differentiellen Umgang der Programmadressaten mit dem Konjunkturprogram m (4.). Nach einer Erläuterung des $m$ ethodischen Vorgehens (5.) werden sodann Ergebnisse der Analy se hinsichtlich der Varianz des Mittelabrufs und wahrscheinlichen Mitnahmeeffekten präsentiert (6.) und abschließend hinsichtlich ihrer Implikationen für generelle Steuerungseffekte von Anreizprogrammen diskutiert (7.).

\section{Das Investitionsprogramm für die Kommunen}

Nach anfänglichem Zögern entschied sich die Bundesregierung zum breiten Einsatz konjunkturpolitischer Instrumente zur Bewältigung der Auswirkungen der globalen Finanzkrise, welche im Herbst 2008 ihren Lauf genommen hatte. Zwischen November 2008 und Januar $2009 \mathrm{w}$ urden in kurzem A bstand zw ei K onjunkturpakete verabschiedet. D iese sollten primär einen kurzfristigen Konjunkturim puls setzen, daneben aber auch Kriterien der Nachhaltigkeit und der Förderung der „Zukunftsfähigkeit“ genügen (vgl. Roos 2009). Das erste K onjunkturpaket $m$ it dem Titel „Beschäftigungssicherung durch Wachstum sstärkung “ förderte Investitionen seitens staatlicher und privater Akteure, entlastete private Haushalte und Unternehm en steuerlich und konkretisierte arbeitsm arktpolitische Maßnahmen, insbesondere die Kurzarbeitsregelungen. Ihm folgte nur w enige Monate später im Januar 2009 das zw eite Paket. Der „Pakt für Beschäftigung und Stabilität in Deutschland zur Sicherung der Arbeitsplätze, Stärkung der Wachstumskräfte und Modernisierung des Landes " umfasste u.a. staatliche Investitionen, Steuer- und Abgabensenkungen, die Förderung der Automobilindustrie („Abwrackprämie“) und weitere arbeitsmarktpolitische Maßnahmen. Im Rahmen des zw eiten Maßnahmenpakets beabsichtigte der Bund $m$ it einer Finanzhilfe für „,besonders bedeutsame Investitionen“ ( 8 ZuInvG) in Höhe von zehn Milliarden Euro die Länder und Kom munen zu zusätzlichen Investitionen anzutreiben. Auf diesem Wege wurde das Kommunale Investitionsprogramm ins Leben gerufen. 
Um die Verteilung der Mittel innerhalb des vorgegebenen Zeitfensters zu gewährleisten, mussten Bund und Länder unter großem Zeitdruck eine Reihe von H indernissen beseitigen: Möglichst kurzfristig galt es zu en tscheiden, wie die Mittel verfassungskonform, dabei aber strategisch sinnvoll im plementiert werden können. Das größte Problem stellte hier die K onformität der V erfahren mit dem Verbot einer direkten Aufgabenübertragung vom Bund auf die Kommunen nach Art. 84 Abs.1 GG dar. Über eine Verwaltungsvereinbarung zwischen Bund und Ländern wurde die Umsetzung des Zukunftsinvestitionsgesetzes (ZuInvG) vereinbart, jedoch nicht vereinheitlicht. Artikel 104b GG wurde 2009 dahingehend geändert, dass , [...] der Bund in außergewöhnlichen Notsituationen auch ohne eigene $G$ esetzgebungskompetenz Finanzhilfen gew ähren können [soll]“ (BMF, Brief an die Finanzm inister der Länder am 23.03.2009). Die Änderung soll die Abgrenzung der Förderbereiche nach dem ZuInvG vereinfachen, um den Kreis der förderfähigen Investitionsvorhaben zu erweitern (ebd.). Im ZuInvG ist eine Förderquote verankert: 75\% des Gesamtvolumens des öffentlichen Finanzie rungsanteils der förderfähigen Kosten eines Landes trägt der Bund und 25\% übernehmen die Länder.

Die ,einfache und verwaltungseffiziente Au sgestaltung“ ( $\$ 8$ ZuInvG) der Mittelvergabe übernahm jedes Land in Eigenregie (vgl. Hammerschmid/Stemmler 2010). H ier drängt sich zwangsläufig die Frage auf, wie die Mittel im distributiven Sinne gerecht unter den Kom munen verteilt werden, so dass alle die gleichen Zugangsmöglichkeiten zu den Bundesmitteln haben. Die Länder entwickelten hier unterschiedliche Modelle. Wesentliche Unterschiede ergeben sich dabei aus dem Anteil von Bundesm itteln an der Gesamtförderung, dem Anteil pauschalierter Mittelzuweisung und dem uns interessierenden Verteilungsverfahren. Bei letzterem finden sich rein pauschalisierte $\mathrm{V}$ erfahren in Brandenburg, Mecklenburg-V orpommern, $\mathrm{N}$ iedersachsen, $\mathrm{N}$ ordrhein-Westfalen und Thüringen; reine A ntragsverfahren finden sich in Bay ern, Bremen und Rheinland-Pfalz. Gemischte V erfahren aus Pauschalen und A ntragsverfahren finden sich schließlich in Baden-Württemberg, Sachsen-A nhalt, Sachsen und Schleswig-Holstein. Im Weiteren konzentrieren wir uns auf das kombinierte Verfahren in Baden-Württemberg.

Für Baden-Württemberg wurden insgesamt Bundesmittel im Umfang von rd. 1,2 Mrd. Euro zur Verfügung gestellt. Dabei sollen 65\% (805 Mio. Euro) auf den Bereich Bildung entfallen, die restlichen 35\% (433 Mio. Euro) waren für Infrastruktur vorgesehen. Die Kommunen erhalten insgesamt 70\% (866 Mio. Euro) und das Land 30\% (371 Mio. Euro) der Mittel. Für die Bildungsinfrastruktur stehen den Gem einden, Landkreisen, Verwaltungsverbänden als Schulträgern und kom munalen Schulverbänden so 499 Mio. Euro zu, die über die Bildungsinfrastrukturpauschale verteilt werden. Für jede einzelne Kom mune wird die Pauschale nach Schülerzahl gem äß der Schulstatistik des Jahres 2007 und der Anzahl der Kinder in Kindertageseinrichtungen (Bemessungsgrundlage ist hier die Kinder- und Jugendhilfestatistik 2008) berechnet. Pro Kind und Schüler erhalten die Zuwendungsem pfänger demzufolge etwa 245 Euro (FM BW 2010a). Für den Schwerpunkt Infrastruktur werden 110 Mio. Euro als Pauschale vergeben, die aus der Einwohnerzahl laut der Bevölkerungsfortschreibung zum 30.6.2008 berechnet wird. Jeder Kom mune stehen daher pro Einwohner rund zehn Euro für Infrastrukturm aßnahmen zur Verfügung. Aus Abbildung 1 wird ersichtlich, dass den Kom munen neben den 110 Mio. Euro aus der Infrastrukturpauschale noch weitere 257 Mio. Euro aus den Bundesmitteln bereitgestellt wurden. Dieser Restbetrag wird den Kommunen in Baden-Württemberg über Fachförderungen zugänglich gemacht. 
Abbildung 1: $\quad$ Mittelverteilung in Baden-Württemberg

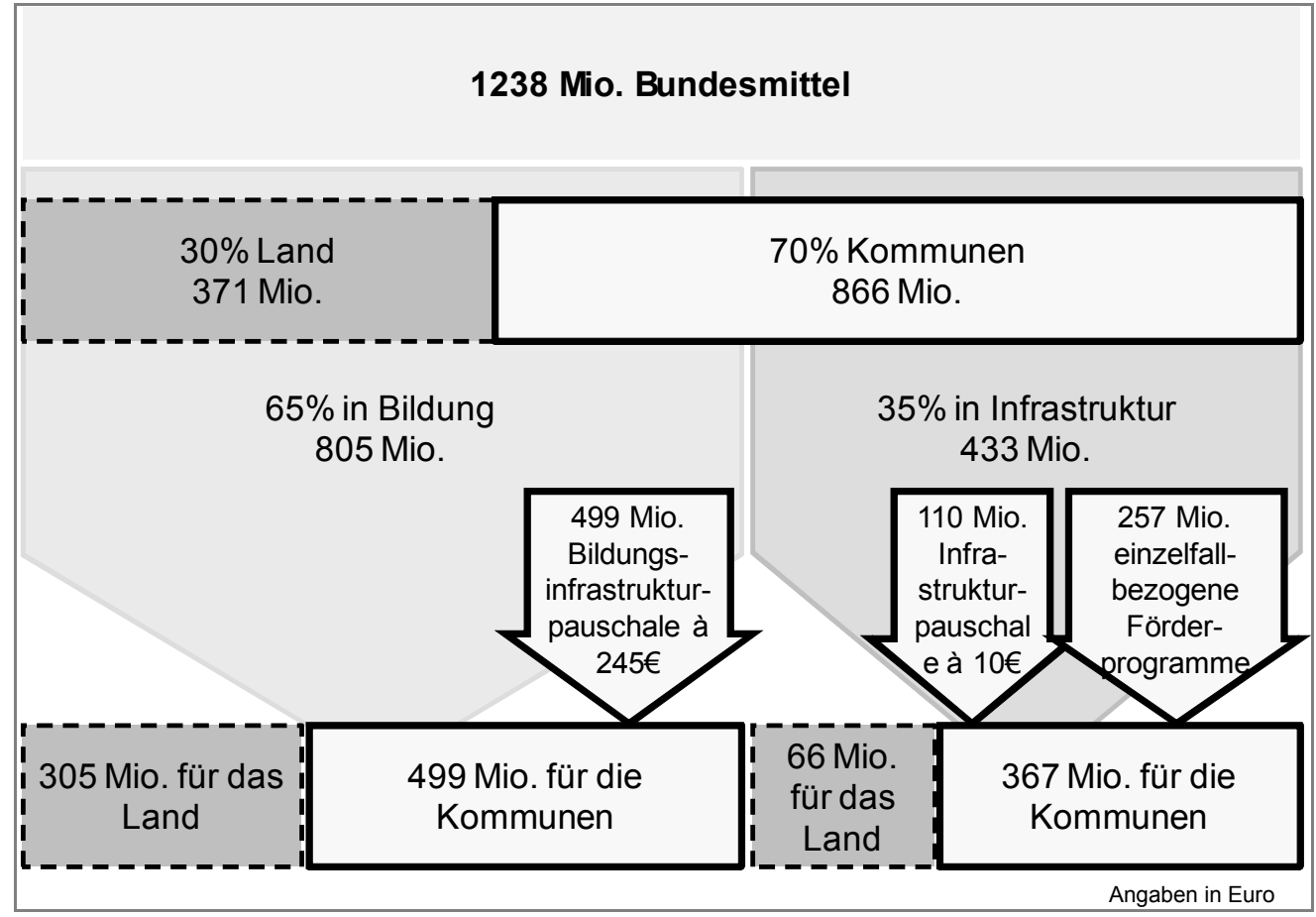

Quelle: Eigene Abbildung nach FM BW 2010b

Die administrative Umsetzung des Zunkunftsinvestitionsprogramms (ZIP) wird in BadenWürttemberg durch ein antragsbasiertes Verfahren gehandhabt, w elches die V ergabe der Bildung- und Infrastrukturpauschale regelt. Die wesentlichen Etappen sind in Abbildung 2 festgehalten.

Abbildung 2: $\quad$ Umsetzungsprozess des ZIP in Baden-Württemberg

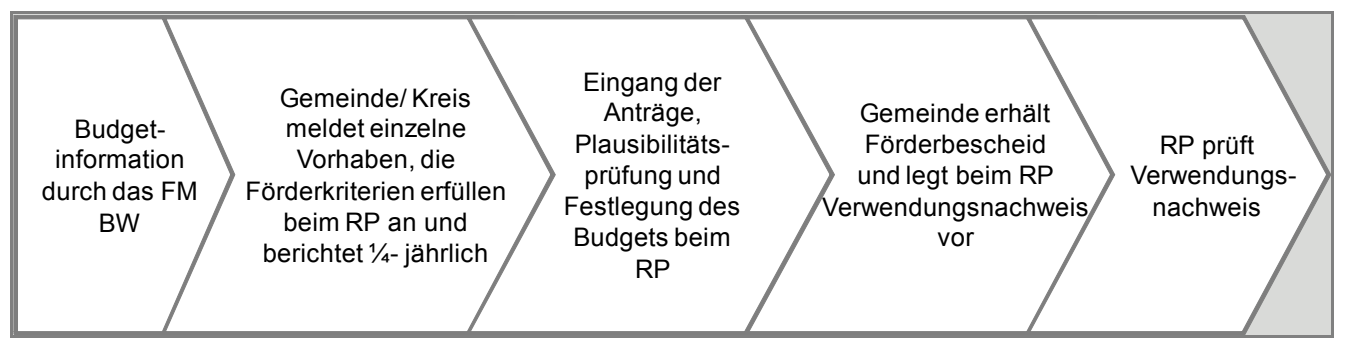

Quelle: Eigene Abbildung nach FM BW 2010d

Das Verfahren ist durch zwei wesentliche Elem ente gekennzeichnet: 1. Die Kommunen müssen selbst aktiv werden, um die Mittel abzuschöpfen. Die Entscheidung, welche Maßnahme beim bewilligenden Regierungspräsidium angemeldet wird, liegt bei den be- 
antragenden Zuw endungsempfängern. Möchten die K ommunen die zur Verfügung gestellten Bundesmittel abrufen, müssen sie einen Eigenanteil von $25 \%$ aufbringen. 2. Die Plausibilitätsprüfungen erfolgen nach einem formalen Verfahren, was angesichts der Menge der Anträge und der K urzfristigkeit des Verfahrens auch nicht anders zu bew erkstelligen gewesen wäre. Um einen tatsächlichen Konjunkturim puls zu setzen ist hier aus Sicht der Programminitiatoren die Zusätzlichkeit der investierten Mittel entscheidend: Es sollten nur Investitionen der Kommunen gefördert werden, die ohne diese Mittel nicht getätigt worden wären. Das bedeutet, dass das V orhaben noch nicht im Haushaltsplan der Kommune festgeschrieben sein darf, $\mathrm{w}$ enn es als $\mathrm{V}$ orschlag beim Regierungspräsidium eingereicht werden soll. Demnach waren Kom munen im Nachteil, die frühzeitig selbst einen Wachstumsimpuls angestrebt haben, indem sie ihre $\mathrm{H}$ aushaltsplanung für das Jahr 2009 früh und fristgem äß abgeschlossen haben. Vorhaben dürfen zudem nicht doppelt, das heißt nicht zugleich durch Mittel aus dem Konjunkturprogramm und durch die anderer Gesetze und Vereinbarungen als auch nicht mit dem KfW-Darlehensprogramm gefördert werden. Vor dem Hintergrund der aktuellen wirtschaftlichen Lage und der finanziellen Situation $\mathrm{m}$ ancher G emeinden (vgl. Holtkamp 2010), ist es für finanziell schwache Gemeinden schw ierig, die Eigenanteile und adm inistrativen K apazitäten aufzubringen, um die für sie bereitgestellten Mittel abzurufen. So ist fraglich, ob die Finanzhilfe bei denjenigen Kommunen ausreichend ankommt, welche sie eigentlich am dringendsten benötigen.

Zusammenfassend lässt sich feststellen, dass die Verteilung der Mittel ein sehr kom plexes Verfahren darstellt, das von den Kom munen zusätzlich zum Tagesgeschäft in einem relativ engen Zeitfenster bewältigt werden $m$ uss. Vergleicht man die Investitionsvolumina der baden-w ürttembergischen $\mathrm{G}$ emeinden, ist eine hohe $\mathrm{V}$ arianz $\mathrm{zw}$ ischen den einzelnen Kommunen festzustellen (vgl. Tabelle 2). Es stellt sich die Frage, wie sich diese Unterschiede in der Höhe der Zukunfts investitionen erklären lassen und ob dam it der Anspruch eines distributiven V erfahrens noch gewährleistet w erden kann. Erstens w ird daher anhand des Investitionsvolum ens die Im plementationseffektivität und zweitens durch die Identifikation von potentiell vorhandenen Mitnahmeeffekten die Steuerungswirkung des untersuchten distributiven Programms untersucht.

\section{Implementationsforschung und distributive Politik}

Die Implementation des Investitionsprogram ms für Kommunen kann weitestgehend als anreizbasierte distributive Policy charakterisiert werden. In der Politikwissenschaft finden sich verschiedene Systematisierungen von Policies (vgl. Windhoff-Héritier 1987). Theodore Lo$w i$ hat mit seiner Unterscheidung von distributiven, regulativen und redistributiven Policies den Grundstein gelegt, um die Natur von Policies als unabhängige Variable zur Erklärung von Politikwirkungen zu verwenden. Nach Lowis These verbinden sich distributive, redistributive und regulative Policies mit je spezifischen Konfliktm ustern und Implementationsproblemen (vgl. Lowi 1972). Redistributive Policies erzeugen so durch Um verteilungseffekte starke Verteilungskonflikte, da Kosten und Nutzen der Program me relativ klar zuzuordnen sind. Regulative Policies erzeugen dagegen ebenso wie distributive Policies durch die weitgehende Gleichverteilung der Be- und Entlastung eher konsensuale Politikmuster. 
Spezifisch sind auch die Im plementationsprobleme: Regulative Politik bringt vor allem Kontrollprobleme und Widerstände der Adressaten mit sich; redistributive Politik ist gegenüber der Gefahr von Moral Hazard und Adverse Selection anfällig und erzeugt so meist hohe administrative Kosten (z.B. zur Bedarfsprüfung); distributive A nreizprogramme sind schließlich w enig zielgenau und bringen Mitnahm eeffekte mit sich (vgl. Bogumil/Jann 2009, S. 176). Die spezifische Konfliktstruktur erklärt die A ttraktivität distributiver Politik als kurzfristiges Instrum ent zur Krisenbewältigung: Da die Kosten und Nutzen breit gestreut sind und es scheinbar keine „, Verlierer" gibt, sind kaum Widerstände und eine relativ schnelle U msetzung zu erw arten: also eine ideale „Simplifizierungsstrategie“ (Klenk/Nullmeier 2010). Auf der anderen Seite sind allerdings auch die Problem e der Gießkannenpolitik voraussehbar: Sie ist wenig treffsicher und lädt zur Mitnahme ein.

Tabelle 1: Die Policy-Typen: Merkmale, Wirkungen und Steuerungsprinzipien

\begin{tabular}{|c|c|c|c|c|}
\hline Policy-Typ & Merkmale der Policy & $\begin{array}{c}\text { Merkmale der } \\
\text { Arena }\end{array}$ & $\begin{array}{l}\text { Steuerungs- } \\
\text { prinzipien }\end{array}$ & Implementationsprobleme \\
\hline Distributiv & $\begin{array}{l}\text { Gleichbehandlung der } \\
\text { Adressaten; Kosten } \\
\text { und Nutzen diffus }\end{array}$ & $\begin{array}{ll} & \text { konsensual } \\
\text { - } & \text { keine Opposition }\end{array}$ & - Anreiz & $\begin{array}{ll}\text { - } & \text { Geringe Zielgenauigkeit } \\
\text { - } & \text { Mitnahmeeffekte }\end{array}$ \\
\hline Re-distributiv & $\begin{array}{l}\text { Relation zw. Kosten } \\
\text { und Nutzen deutlich } \\
\text { (umverteilend) }\end{array}$ & $\begin{array}{ll}- & \text { Konfliktorientiert } \\
\text { - } & \text { Polarisierung zw. } \\
& \text { Gewinnern/Ver- } \\
& \text { lierern } \\
\text { - } & \text { Ideologische Unter- } \\
& \text { mauerung }\end{array}$ & $\begin{array}{l}\text { - Staatlicher } \\
\text { Zwang } \\
\text { - Sozial selektive } \\
\text { Leistungs- } \\
\text { gewährung/ } \\
\text {-zuweisung }\end{array}$ & $\begin{array}{l}\text { - } \text { Umgehungsstrategien } \\
\text { - Großer bürokratischer } \\
\text { Aufwand } \\
\text { - } \quad \text { Politisierung } \\
\text { - } \text { Moral Hazard und Adver- } \\
\text { se Selection }\end{array}$ \\
\hline Regulativ & $\begin{array}{l}\text { Verhaltensvorschriften } \\
\text { für private Aktivitäten }\end{array}$ & $\begin{array}{l}\text { Wechselnde Koali- } \\
\text { tionen und Konflikt- } \\
\text { linien } \\
\text { - Je nach Kosten/Nut- } \\
\text { zen unterschiedliche } \\
\text { Organisationen be- } \\
\text { troffen }\end{array}$ & $\begin{array}{ll}\text { - Staatlicher } \\
\text { Zwang (Gebot/ } \\
\text { Verbot) } \\
\text { - Überzeugung } \\
\text { und Vorbild } \\
\text { - Selbstregulation } \\
\text { durch Betroffene }\end{array}$ & $\begin{array}{l}\text { Vollzugs- und Kontroll- } \\
\text { defizite } \\
\text { - Widerstände der Adres- } \\
\text { saten }\end{array}$ \\
\hline
\end{tabular}

Eigene Darstellung auf Basis von Lowi 1972; Heinelt 2009; Hill/Hupe 2009

Im Rahmen des Zukunftsinvestitionsgesetzes war die programmgemäß gleichberechtigte Verteilung der Mittel zunächst nur ein Nebenziel, prim äres Ziel blieb die Setzung von Konjunkturimpulsen bei gleichzeitiger Verfolgung von Nachhaltigkeitszielen. Eine Ungleichverteilung der Mittel würde jedoch auch dieses Program mziel in Frage stellen, wenn damit ein V erfehlen des Zusätzlichkeitskriterium s einherginge. D amit eröffnet das Konjunkturprogramm einen hervorragenden Testfall zur $U$ ntersuchung klassischer Im plementationsprobleme distributiver Policies, namentlich der Ungleichverteilung der Mittel und die Entstehung von Mitnahmeeffekten.

Ungleichverteilung (oder in der Sprache der frühen Implementationsforschung „Soziale Selektivitäten“ (Mayntz 1983)) beschreibt hier A bweichungen vom distributiven Verteilungsziel, die nicht zufällig gestreut sind und auffällige Muster aufweisen. Im ersten Schritt der quantitativen Analy se ist daher das Investitionsvolum en der Kommunen in Baden-Württemberg im Rahmen des Kommunalen Investitionsprogramms der Bundesregierung die abhängige $\mathrm{V}$ ariable. Die erfolgreiche Im plementation des Kommunalen Investitionsprogramms wird so definiert, dass di e Mittelverteilung um so effektiver ist, je 
einheitlicher die Zugangschancen der Zuwendungsem pfänger zu den Mitteln sind. Zwar haben alle Kommunen Baden-Württembergs theoretisch gesehen die gleichen Möglichkeiten die für sie vorgesehenen Mittel abzurufen, jedoch lässt die Varianz der Investitionsvolumina der einzelnen Kommunen vermuten, dass die praktischen Chancen divergieren.

Mitnahmeeffekte bezeichnen hier die Inanspruchnahm e finanzieller Anreize als Belohnung für ein V erhalten, das auch ohne den zusätzlichen A nreiz stattgefunden hätte. Damit wird die Lenkungswirkung konterkariert. Da sich das jeweilige Ausmaß eines Mitnahmeeffekts empirisch nicht exakt ermitteln, sondern nur verm uten lässt, ist die Bewertung des Effekts umstritten. Ziel der folgenden Analyse ist es, Ursachen für die auftretende Varianz und etwaige Mitnahmeeffekte aufzudecken (vgl. auch Hill/Hupe 2009, S. 77).

\section{Erklärungsfaktoren für Ungleichverteilung und Mitnahmeeffekte}

Die Implementationsforschung hat sich zwar mittlerweile vom Ziel anspruchsvoller theoretischer Modelle verabschiedet (vgl. Knill/Tosun 2010; DeLeon/DeLeon 2002; Hill/Hupe 2009); im Rahmen von Ansätzen m ittlerer Reichweite haben sich inzwischen jedoch mehrere Bündel von $\mathrm{K}$ ausalbegründungen der A bweichung von Policies von ihren Programmzielen ausdifferenziert. Im Einzelnen werden hier Programmdefizite, Kontrolldefizite, Inform ationsasymmetrien, Problem e der Instrum entenwahl, fehlende institutionelle Kompatibilitäten, Präferenzen und Strategien der im plementierenden Akteure sowie die administrative Kapazität der im plementierenden Ebene genannt (vgl. Knill/Tosun 2010, S. 121-131). Aus diesem Bündel möglicher Ursachen lassen sich im Weiteren zur Bildung von $\mathrm{H}$ ypothesen zur differentiellen $\mathrm{U}$ msetzung des Konjunkturprogrammes zwei wesentliche Aspekte herausgreifen: Charakte ristiken des Program ms einerseits und Eigeninteressen, Strategien und Kapazitäten der Adressaten andererseits. Dabei bilden Programmdefizite, Informationsasymmetrien und Instrum entenwahl den (für alle Adressaten konstanten) Rahmen, in dem - so unsere These - spezifische Präferenzen und Strategien der Akteure sowie deren administrative Kapazität die wesentlichen Unterschiede erklären können. Im Weiteren w erden Hypothesen zur differentiellen Inanspruchnahme des Anreizprogramms skizziert. Da der program matische Rahmen stabil bleibt, werden zur Erklärung der differentiellen A usnutzung der so eröffneten Im plementationsspielräume durch die K ommunen als Program madressaten im Folgenden Problem druck, administrative Kapazitäten und politische Präferenzen als wesentliche Einflussfaktoren betrachtet.

\subsection{Ungleichverteilung im Investitionsvolumen}

\section{Problemdruck}

Die Haushaltslage der baden-württembergischen Kommunen ist im Vergleich zu anderen Bundesländern zwar weniger drückend (vgl. Holtkamp 2010), dennoch spielen auch hier Haushaltsrestriktionen eine entscheidende Rolle für die Politikgestaltung. Zunächst ließe sich annehmen, dass finanzschwache Kom munen aus purer Not stärker auf Mittel des 
Konjunkturprogramms zurückgreifen. Eine Reihe von Program mcharakteristiken sorgt jedoch dafür, dass die vermuteten Auswirkungen umgekehrt sein sollten. Zunächst ist da die Eigenbeteiligung: So ist zu erw arten, dass die $\mathrm{H}$ aushaltslage eine Auswirkung darauf hat, ob und $\mathrm{w}$ ie viele $\mathrm{V}$ orhaben eine Kommune beim zuständigen Regierungspräsidium anmeldet, da der Eigenanteil von 25\% für finanzschw ache Kommunen eine $\mathrm{H}$ ürde darstellen kann. Um dieses Problem aufzuf angen hat das Land Baden-Württem berg den Ausgleichsstock um 60 Mio. Euro erhöht, sodass für die als finanzschwach eingestuften Kommunen insgesamt 210 Mio. Euro als A usgleichsstock zur Beantragung zur Verfügung stehen ( $\mathrm{VwV}$ Ausgleichsstock). Setzt $\mathrm{m}$ an also rationales Handeln der Kom munen voraus, werden sie nur Mittel aus dem Konjunkturpaket beantragen, wenn sie in der Lage sind den Eigenanteil von $25 \%$ selbst oder über den A usgleichsstock zu finanzieren. A us diesem theoretischen Zusammenhang lässt sich folgende Hypothese ableiten:

H1: Je besser ihre Haushaltslage, desto höher das Investitionsvolumen der Котmunen.

\section{Administrative Kapazität}

Antragsbasierte Verfahren, wie sie in zahlreichen an die K ommunen gerichteten Programmen die Regel sind, setzen eine adm inistrative Kapazität zur Antragstellung voraus (vgl. Bogumil/Grohs/Reiter 2008). A dministrative K apazität auf kommunaler Ebene hängt dabei vor allem von der Größe der Kommune wie von deren Personalausstattung ab (vgl. auch Bogumil et al. 2007, S. 97ff.). D ie Spannbreiten sind hier erheblich und reichen in Baden-Württemberg von Kleinstgemeinden mit weniger als fünf Mitarbeitern zu Großstadtverwaltungen mit über 17.000 Mitarbeitern. K lein- und Kleinstgemeinden verfügen so kaum über die notw endigen Ressourcen, um über das „Tagesgeschäft" hinausgehende Aktivitäten und eine Einarbeitung in das A ntragsprozedere leisten zu können. Demgegenüber verfügen Großstadtverwaltungen häufig über spezialisierte V erwaltungsakteure, die das „Antragsgeschäft“ beherrschen. Wir nehm en daher an, dass K ommunen mit größerer Verwaltungskapazität eher in der Lage sind, A nträge innerhalb kurzer Zeit vorzubereiten und zu stellen, als $\mathrm{K}$ ommunen $\mathrm{m}$ it einer geringen $\mathrm{V}$ erwaltungskapazität, die den zusätzlichen Organisationsaufwand auf w eniger spezialisierte Mitarbeiter verteilen müssen.

\section{H2: Je größer die Kommune, desto höher ist ihr Investitionsvolumen.}

H3: Je mehr Personal je Einwohner eine Kommune beschäftigt, desto höher ihr Investitionsvolumen.

\section{Parteipolitische Präferenzen}

Ein dritter zu testender Einflussfaktor auf das Investitionsvolumen der Kommunen ist die Zusammensetzung des Entscheidungsorgans, das über das Etatrecht verfügt. D er Parteiendifferenzhypothese von Hibbs (1977) folgend kann erw artet werden, dass eine bestimmte parteipolitische Färbung des Rates Auswirkungen auf die Höhe des Investitionsvolumens hat. Konkret steht zu verm uten, dass rechte Parteien eine die Marktkräfte stärkende Politik verfolgen, während linke Parteien eher gewillt sind in die Volkswirtschaft 
einzugreifen und eine ausgabenorientierte Politik zu verfolgen (ebd.). Kontrazy klische Konjunkturpolitik ist zudem ein typisches Instrument keynesianischer Politik, die größere Unterstützung bei sozialdemokratischen Parteien findet. Daher gehen wir - ähnlich Wagschal und Jäckel (2010, S. 311), davon aus, dass das Investitionsvolum en in Kommunen mit hohem Anteil linker bzw. sozialdemokratischer Sitze im Rat höher ist:

\section{H4: Je mehr Sitze im Rat der Kommune an linke und sozialdemokratische Parteien vergeben sind, desto höher ist ihr Investitionsvolumen.}

Aus der lokalen Politikforschung wissen wir, dass der Grad des Parteienwettbewerbs und damit der Einfluss parteipolitischer Variablen zwischen unterschiedlichen Gem eindeordnungen und unterschiedlichen $\mathrm{G}$ emeindegrößen stark variiert (vgl. Holtkamp 2008). D aher gehen wir ergänzend davon aus, dass der Einfluss der parteipolitischen Variablen mit abnehmender Gemeindegröße abnimmt, da in kleinen Gemeinden eher konkordante Politikmuster zu erwarten sind.

H4a: Der Effekt linker und sozialdemokratischer Parteien auf das Investitionsvolumen reduziert sich mit abnehmender Gemeindegröße.

\subsection{Mitnahmeeffekte}

Im zweiten Schritt der Analyse gilt es zu prüfen, ob bei der Distribution der Mittel Mitnahmeeffekte seitens der Kommunen auftreten. D arüber hinaus soll getestet w erden, ob die oben genannten Einflussfaktoren (kom munale Schulden, Verwaltungskapazität, Zusammensetzung des Rates und $\mathrm{G}$ röße) Mitnahm eeffekte begünstigen, um zu ergründen, welche Kommunen stärker oder schwächer von der distributiven Politik profitieren. Ein Mitnahmeeffekt ist nach der hier verw endeten D efinition dann vorhanden, wenn eine Kommune Mittel aus dem Kom munalen Investitionsprogramm abruft, aber insgesamt keine höheren Investitionen vorweisen kann. Au s den zuvor genannten theoretischen Erklärungen der unabhängigen $\mathrm{V}$ ariablen ergeben sich bezüglich Mitnahmeeffekten daher folgende Annahmen:

\section{Problemdruck}

Zwar müsste man annehmen dass ein stärkerer haushaltspolitischer Druck die Kommunen zur verstärkten Nutzung von Mitnahm eeffekten treibt. Jedoch w ird dieser Effekt durch abnehmende $\mathrm{H}$ andlungsspielräume letztendlich aufgehoben. Daher wird angenommen, dass Kommunen mit schlechter Haushaltslage weniger dazu in der Lage sind „, mitzunehmen". Der Eigenanteil ist für w eniger verschuldete Kommunen einfacher und unkom plizierter zu leisten, was sie somit dazu bewegen kann mehr Projektanträge für Fördergelder zu stellen.

H5: Je schwächer die Haushaltslage der Kommunen, desto unwahrscheinlicher sind die Mitnahmeeffekte. 


\section{Administrative Kapazität}

Analog zum Investitionsvolumen gehen wir davon aus, dass die administrative Kapazität Einfluss auf die Mitnahm eeffekte hat. Wie im Zusammenhang des Investitionsvolum ens als abhängige Variable, beeinflusst die Gemeindegröße die V erwaltungskapazität ebenso wie der relative Personalbestand.

H6: Je größer die Kommune, desto wahrscheinlicher sind die Mitnahmeeffekte.

H7: Je mehr Personal je Einwohner eine Kommune beschäftigt, desto stärker sind die Mitnahmeeffekte.

\section{Parteipolitische Präferenzen}

Die parteipolitischen Annahmen bezüglich der Mitnahmeeffekte werden aus den theoretischen Ausführungen zum Einfluss der Parteien auf das Investitionsvolum en abgeleitet. Nimmt man an, dass sozial-dem okratische Parteien eher zu konjunkturpolitischen Maßnahmen greifen, ist davon auszugehen, dass ein höherer A nteil links-sozialdem okratischer Parteien mit stärkeren Mitnahmeeffekten einhergeht.

H8: Je mehr Sitze im Entscheidungsorgan der Kommune an linke und sozialdemokratische Parteien vergeben sind, desto stärker sind die Mitnahmeeffekte.

Diese Hypothese muss wiederum dahingehend spezifiziert werden, dass die konkordanteren Orientierungen in kleinen Gemeinden den Parteiwettbewerb eindämmen.

H8a: Der Effekt linker und sozialdemokratischer Parteien auf die Höhe der Mitnahmeeffekte reduziert sich mit abnehmender Gemeindegröße.

\section{Methodisches Vorgehen}

Die w eitere Untersuchung basiert auf einer $\mathrm{V}$ ollerhebung unter den baden-württembergischen K ommunen. Für die ausschließliche Betrachtung Baden-Württem bergs spricht, dass bis Mitte 2009 bereits ein großer Anteil des den Bundesländern gem äß ZuInvG verfügbaren Investitionsbudgets in nachvollziehbaren Vorhaben- oder Projektlisten dokumentiert und somit die Mittelverwendung bereits sehr weit fortgeschritten war (Handrich et al. 2009, S. 750). Für die bis heute noch nicht vollständig abgeschlossene Im plementation der distributiven Maßnahm en ist daher im Bundesländervergleich eine überdurchschnittlich genaue Messung möglich.

Bei der Operationalisierung w urde folgendermaßen vorgegangen: Zur Messung des Investitionsvolumens wurde die „Vorhabenliste der bewilligten Projekte des Zukunftsinvestitionsprogramms" zum Stichtag 23.06.2010 herangezogen, die vom Finanzministerium Baden-Württemberg zur Verfügung gestellt wurde. Als Bezugsgröße wurden nicht die pauschalverteilten Mittel, sondern nur die über förderfähige Projekte verteilten Mittel (insgesamt 257 Millionen Euro) herangezogen. Hierzu wurde der über die Infrastrukturpauschale und Bildungspauschale errechnete Bundesanteil von der $\mathrm{G}$ esamtsumme pro Kommune abgezogen. D ie Gemeindegröße wurde über den Einw ohnerstand zum 30.06. 
2008 gemessen (Statistische Ä mter des Bundes und der Länder 2009). Zur Messung der Haushaltslage wurde der kommunale Schuldenstand des Jahres 2008 (Statistisches Landesamt Baden-Württemberg) verwendet. Die Verwaltungskapazität wird durch den Q uotienten Personal pro Einw ohner im Jahr 2008 operationalisiert (Personalstandsstatistik des Statistischen Landesamtes Baden-Württemberg). Bei der A nalyse der Zusammensetzung des Rates wurden die Sitzanteile in den Gemeinderäten der CDU, der FDP und der FW als bürgerliche liberale V ariable (C DU_FDP_FW) addiert und die entsprechenden Anteile der SPD und der G RÜNEN als links-sozialdem okratische Variable (SPD_GRÜNE).

Die größten m ethodischen Probleme ergeben sich bei der Messung der Mitnahmeeffekte. Mitnahmeeffekte beruhen auf verdecktem Handeln, das sich nicht direkt nachweisen lässt. A us diesem Grund versuchen w ir die Wahrscheinlichkeit von Mitnahmeeffekten über den Umweg des Investitionsverhaltens der Kommunen über die zurückliegenden fünf Jahre anzugehen. Dazu wurde der Du rchschnitt der Investitionsvolumina der Kom munen aus den Jahren 2004 bis 2008 berechnet. Dieser Durchschnittswert dient als Referenzwert. Da Investitionen jedoch nicht nur von verfügbaren Zuschüssen abhängen, sondern von der allgem einen Einnahm esituation einer $\mathrm{K}$ ommune, müssen zusätzlich konjunkturelle und durch Ä nderungen des Steuerrechts bedingte V eränderungen der Steuereinnahmen berücksichtigt werden. Hierzu wurde ein Prozentsatz (P) berechnet, um welchen die durchschnittlichen Steuereinnahm en der Jahre 2004 bis 2008 hinter denen des Jahres 2009 liegen. Der Referenzwert wurd e auf der Basis dieser Abweichung anteilig verringert, um die Mitnahm eeffekte zu berechnen. D abei w ird angenommen, dass Mitnahmeeffekte per definitionem dann vorhanden sind, wenn das Investitionsvolumen einer Kommune im Jahr 2009 nicht höher liegt, als der festgesetzte Referenzwert der Jahre 2004 bis 2008 verringert um den Steuerprozentanteil P. Inhaltlich bedeutet dies, dass keine zusätzlichen Investitionen, auch nicht durch das Kom munale Investitionsprogram m, getätigt wurden. Die so berechnete Variable Mitnahmeeffekte aus der Differenz zwischen dem Referenzwert und den Investitionen des Jahres 2009 stellt som it eine Proxy variable für die Mitnahm eeffekte dar und spiegelt inhaltlich die Zusätzlichkeit der Investitionen wider. Die Daten zu eben genannten Berechnungen w urden auf N achfrage vom Statistischen Landesamt Baden-Württemberg zur Verfügung gestellt und entstammen der Kommunalen Jahresrechnungsstatistik der Jahre 2004 bis 2008 und 2009.

\section{Analyse}

Sowohl für das Investitionsvolum en (Analyseschritt 1) als auch für die Mitnahm eeffekte (Analyseschritt 2) als abhängige Variable wu rden die Tests jeweils zweistufig durchgeführt. Zunächst wurde der bivariate Zusammenhang geprüft. Danach wurden zur Identifikation plausibler Erklärungsmodelle lineare Regressionsmodelle eingesetzt.

Tabelle 2 präsentiert die Mittelwerte sowi e die Spannweite des Investitionsvolum ens für die verschiedenen $\mathrm{G}$ emeindegrößenklassen. Insgesam t w urden Zuw endungen für 4.472 Einzelm aßnahmen $\mathrm{m}$ it einem Investiti onsvolumen von 1,07 Mrd. Euro bewilligt. Nach Angaben des Finanzm inisteriums verzichteten lediglich $23 \mathrm{~K}$ ommunen in BadenWürttemberg auf Zuw endungen (vgl. Rechungshof Baden-Württem berg 2011). Ersicht- 
lich wird hierbei, dass zwar generell die G röße der K ommune erheblichen Einfluss auf die Investitionsvolum ina hat, aber erhebliche Varianz zwischen einzelnen Kom munen ähnlicher Größe besteht. So liegt das Maximum der G K4 weit über dem Minimum der GK3, was die Annahm e berechtigt, dass das Investitionsvolum en nicht einzig von der Gemeindegröße abhängig ist.

Tabelle 2: $\quad$ Mittelwerte des Investitionsvolumens je Gemeindegrößenklasse

\begin{tabular}{lrrrrrr}
\hline & Fälle & \multicolumn{1}{c}{$\begin{array}{c}\text { Mittelwert } \\
\text { (in €) }\end{array}$} & $\begin{array}{l}\text { Mittelwert } \\
\text { pro Kopf } \\
\text { (in } € \text { ) }\end{array}$ & \multicolumn{1}{c}{$\begin{array}{c}\text { Min } \\
\text { (in } € \text { ) }\end{array}$} & \multicolumn{1}{c}{$\begin{array}{c}\text { Max } \\
\text { (in } € \text { ) }\end{array}$} & Std. Abw. \\
\hline$>400.000$ & 1 & 82.100 .000 & 136,72 & 82.100 .000 & 82.100 .000 & .5 \\
$200.000-400.000$ & 3 & 53.700 .000 & $\mathbf{2 0 8 , 6 8}$ & 42.900 .000 & 73.500 .000 & 17.200 .000 \\
$100.000-200.000$ & 5 & 26.400 .000 & 199,21 & 3.808 .107 & 73.400 .000 & 29.100 .000 \\
$50.000-100.000$ & 13 & 13.300 .000 & 171,03 & 1.818 .422 & 66.700 .000 & 18.400 .000 \\
$25.000-50.000$ & 50 & 2.756 .237 & $\mathbf{7 8 , 9 4}$ & -27.874 & 17.100 .000 & 3.041 .573 \\
$10.000-25.000$ & 175 & 1.316 .986 & 83,31 & -691.714 & 15.400 .000 & 2.077 .383 \\
$<10.000$ & 848 & 361.196 & 106,02 & -412.010 & $\mathbf{7 . 9 5 1 . 4 6 2}$ & 625.567 \\
\hline insgesamt & 1095 & 1.116 .491 & 102,66 & -691.714 & 82.100 .000 & 5.252 .985 \\
\hline
\end{tabular}

Quelle: Eigene Darstellung Anmerkung: N egative Minima des Investitionsvolumens in Tabelle 2 entstehen durch die Bestimmung des Investitionsvolumens nach Abzug der Pauschalen. Hat eine Kommune die Pauschalen noch nicht vollständig abgerufen, entsteht ein negatives Investitionsvolumen I.

Wahrscheinliche Mitnahmeeffekte nach unserer Definition konnten in insgesamt 456 Fällen, also 41,6\% nachgewiesen werden. Tabelle 3 zeigt das Vorliegen von Mitnahmeeffekten nach Größenklassen getrennt auf.

Tabelle 3: Anteil der Kommunen die Mitnahmeeffekte aufweisen

\begin{tabular}{lccc}
\hline & 1095 Kommunen & davon $\mathbf{4 5 6}$ mit ME & Anteil an allen Kommunen \\
\hline GK1-3 & 9 & 4 & $44,44 \%$ \\
GK 4 & 13 & 7 & $53,84 \%$ \\
GK 5 & 50 & 24 & $48,00 \%$ \\
GK 6 & 175 & 81 & $46,28 \%$ \\
GK 7 & 848 & 340 & $40,09 \%$ \\
\hline
\end{tabular}

Quelle: Eigene Tabelle.

Es zeigt sich hier also für beide betrachteten Sachverhalte eine deutliche Varianz, deren Aufklärung nicht allein über den nahe liegenden Faktor Größe erfolgen kann. Daher werden im Folgenden die in Abschnitt 5 skizzierten Hy pothesen zur weiteren Analyse herangezogen.

\subsection{Varianz des Investitionsvolumens}

In einer bivariaten A nalyse ergeben sich für die meisten Einflussvariablen nur teilweise die erwarteten Zusam menhänge. Sowohl für di e Größe wie für (in allerdings deutlich schwächerem Maße) die Verwaltungskapazität gelten die erwarteten positiven Zusam menhänge. Abweichungen ergeben sich hingegen hinsichtlich der Verschuldung wo die Effektrichtung nicht wie erwartet negativ, s ondern positiv ist. Dies würde bedeuten, dass 
mit Anstieg der Schulden das Investitionsvolum en der Kom munen steigt. Die Zusammenhänge hinsichtlich der parteipolitischen Variablen stellen sich in der bivariaten Analyse wie erwartet dar: Während die bürgerlich-liberalen Parteien einen leicht negativen Einfluss auf das Investitionsvolumen haben, ist bei dominanten linken Parteien ein positiver Einfluss zu vermelden.

Tabelle 4: Bivariate Regression- Investitionsvolumen als abhängige Variable

\begin{tabular}{|c|c|c|c|c|c|}
\hline Investitionsvolumen & Modell 1 & Modell 2 & Modell 3 & Modell 4 & Modell 5 \\
\hline \multicolumn{6}{|l|}{ Unabhängige Variablen } \\
\hline Größe & $\begin{array}{c}0,808^{* * *} \\
(3,571)\end{array}$ & & & & \\
\hline Schulden & & $\begin{array}{c}0,7064^{* * *} \\
(0,0026)\end{array}$ & & & \\
\hline Verwaltungskapazität & & & $\begin{array}{c}0,2661^{* * *} \\
(3,96 \mathrm{e}+07)\end{array}$ & & \\
\hline Bürgerlich-liberale & & & & $-0,0526^{*}$ & \\
\hline Parteien & & & & $(569501,3)$ & \\
\hline Sozial-demokratische & & & & & $0,1829^{* * *}$ \\
\hline Parteien & & & & & $(1111303)$ \\
\hline $\bar{N}$ & 1095 & 1095 & 1095 & 1095 & 1095 \\
\hline Adj. $R^{2}$ & .6519 & .4985 & .0699 & .0019 & .0326 \\
\hline
\end{tabular}

Anmerkungen: ${ }^{* * *} \mathrm{p}<0,01,{ }^{* *} \mathrm{p}<0,05,{ }^{*} \mathrm{p}<0,1$. Angegeben ist der standardisierte Regressionskoeffizient. Standardfehler in Klammern. VIF Werte zeigen keinen Hinweis auf Multikollinearität.

Führt $\mathrm{m}$ an diese Erklärungsfaktoren in einem $\mathrm{m}$ ultiplen Regressionsm odell zusam men (vgl. Tabelle 5), wird zunächst deutlich (Modell 6), dass der standardisierte Regressionskoeffizient, der zum Vergleich der Einflussst ärke der unterschiedlichen Variablen herangezogen wird, bei der Größe im multiplen Modell mit b*=0,9382 einen größeren Effekt aufweist, als im bivariaten Modell. Markante V eränderungen im Vergleich zur bivariaten Analyse gibt es vor allem beim Prädiktor Schulden. Er ist noch immer signifikant, weist nun jedoch eine andere Effektrichtung auf. Das heißt in dem Fall, dass das Investitionsvolumen bei Schuldenanstieg einer K ommune sinkt. D ie theoretische A nnahme, dass bei höheren Schulden ein geringeres Investitionsvolum en vorhanden sein könnte, wird unter Kontrolle der Größe und der anderen unabhängigen V ariablen folglich in Modell 6 bestätigt. Ein Einfluss bürgerlich-liberaler Partei en auf das Investitionsvolum en der Gemeinden kann $\mathrm{m}$ it Modell 6 nicht $\mathrm{m}$ ehr nachgew iesen werden. Die zweite Parteienvariable (SPD_GRÜNE) ist nach wie vor hoch signifikant; mit einem $b^{*}=-0,0686$ hat sich jedoch auch hier die Einflussrichtung ins N egative umgekehrt. Bei einem Anstieg des Sitzanteils der SPD und Grünen im Gemeinderat geht folglich das Investitionsvolumen zurück. 
Tabelle 5: $\quad$ Multiple Regression nach Gemeindegrößenklasse

\begin{tabular}{|c|c|c|c|c|c|c|}
\hline Investitionsvolumen M & $\begin{array}{l}\text { odell } 6 \\
\text { Alle }\end{array}$ & $\begin{array}{l}\text { Modell } 7 \\
\text { GK1-GK3 }\end{array}$ & $\begin{array}{c}\text { Modell } 8 \\
\text { GK4 }\end{array}$ & $\begin{array}{l}\text { Modell } 9 \\
\text { GK5 }\end{array}$ & $\begin{array}{l}\text { Modell } 10 \\
\text { GK6 }\end{array}$ & $\begin{array}{c}\text { Modell } 11 \\
\text { GK7 }\end{array}$ \\
\hline \multicolumn{7}{|l|}{ Unabhängige Variablen } \\
\hline Größe & $\begin{array}{c}0,9381 \\
(8,3360)\end{array}$ & $\begin{array}{c}1,2782 \\
(186,36)\end{array}$ & $\begin{array}{c}0,6750 \\
(320,77)\end{array}$ & $\begin{array}{l}0,1010 \\
(61,96)\end{array}$ & $\begin{array}{l}0,2616 \\
(44,41)\end{array}$ & $\begin{array}{l}0,1782 \\
(12,18)\end{array}$ \\
\hline & $-0,1090$ & $-0,3035$ & $-0,3178$ & 0,1595 & 0,1951 & 0,1266 \\
\hline Schulden & $(0,0048)$ & $(0,0553)$ & $(0,1156)$ & $(0,0230)$ & $(0,0164)$ & $(0,0071)$ \\
\hline Venwaltungskanazität & $-0,0337$ & $-0,5206$ & $-0,1988$ & $-0,0554$ & 0,0420 & 0,0426 \\
\hline verwaltungskapazıtat & $(2,64 \mathrm{e}+07)$ & $(5,49 \mathrm{e}+09)$ & $(7,85 e+08)$ & $(1,57 e+08)$ & $(4,36 e+07)$ & (6492437) \\
\hline \multirow{2}{*}{ Sozial-demokratische Parteien } & 0,0658 & 0,0464 & $-0,2153$ & $-0,1288$ & $-0,1208$ & $-0,0660$ \\
\hline & $(707899,2)$ & $(1,33 e+08)$ & $(6,28 \mathrm{e}+07)$ & $(5746394,0)$ & $(1310384,0)$ & $(181736,6)$ \\
\hline$\overline{\mathrm{N}}$ & 1095 & 9 & 13 & 50 & 175 & 848 \\
\hline Adj. $R^{2}$ & 0,6117 & & & & & \\
\hline
\end{tabular}

Anmerkungen: Angegeben ist der standardisierte Regressionskoeffizient, sow ie der Standardfehler. Alle Modelle wurden negativ auf Multikollinearität getestet. Der standardisierte Regressionskoeffizient in Modell 7 ist vermutlich aufgrund der geringen Fallzahl in diesem Modell größer 1; Auf die Angabe des Adj. $\mathrm{R}^{2}$ wird bei den Messungen nach GK verzichtet, da mit zunehmender Fall zahl das Adj.R² steigt und damit folglich kein verlässlicher Modellvergleich möglich ist. Die Eint eilung der Gemeindegrößenklassen ist wie folgt: GK1= $>400.000 \mathrm{EW}, \mathrm{GK} 2=200.000-400.000 \mathrm{EW}, \mathrm{GK} 3=100 . \quad 000-200.000 \mathrm{EW}, \mathrm{GK} 4=50.000-100.000 \mathrm{EW}, \mathrm{GK} 5=$ 25.000-50.000EW, GK6=10.000-25.000EW, GK7=<10.000EW .

Bereits bei der Betrachtung der Daten mittels der deskriptiven Statistik wurde deutlich, dass die Größe einen starken Einfluss auf da s Investitionsvolumen hat, da m eist einwohnerstarke Kommunen ein relativ hohes Investitionsvolum en (sowohl absolut als auch pro Kopf) erkennen ließen. Unter Kontrolle aller anderen genannten unabhängigen V ariablen bestätigte sich diese Plausibilitätsannahm e au ch in der Regressionsanaly se. Der für die bivariate Regression festgestellte Einfluss der unabhängigen Variablen auf das Investitionsvolumen erwies sich auch unter K ontrolle der jew eils anderen $\mathrm{V}$ ariablen als signifikant. Unter Berücksichtigung der Besonderheiten, die sich für die Analyse von Vollerhebungen ergeben (vgl. Behnke 2005), steht in der weiteren Analyse jedoch nicht die Signifikanz, sondern die Relevanz der Prädiktoren für die Erklärung der Varianz des Investitionsvolumens im Mittelpunkt des Interesses. Um die Effekte des extrem en Größenunterschiedes der Gemeinden möglichst vergleichbar zu untersuchen, w urde die Regressionsanalyse für die verschiedenen $\mathrm{G}$ emeindegrößenklassen (Modelle 7 bis 11) wiederholt. Dabei war ein Ergebnis, dass auch innerhalb der Gruppen die Größe einen positiven Einfluss auf das Investitionsvolumen ausübt.

Für die GK1-3 und GK4 bestätigt sich die Gegenhypothese der H1: Wenn eine Kommune mehr Schulden verzeichnet, hat sie ein niedrigeres Investitionsvolum en. Der Einfluss der V erwaltungskapazität zeigt hingegen nur für die GK6 und GK7 den erwarteten positiven Einfluss auf das Investitionsvolum en. Für Kom munen, die m ehr als 25.000 Einwohner aufweisen, ist der Effekt hingegen negativ: Je höher die V erwaltungskapazität, desto geringer das Investitionsvolumen. Dies mag mit Sättigungseffekten und Verzerrungen auf Grund zusätzlicher personalintensiver V erwaltungsaufgaben zu erklären sein. Die Parteidifferenzhypothese wird nur für die G roßstädte bestätigt. Umgekehrt verhält es sich für kleine und m ittlere Gem eindegrößen: je m ehr Sitzanteile sozial-dem okratische Parteien im kom munalen Rat haben, desto niedriger fällt das Investitionsvolumen aus. Nur bei den neun größten Städten wirken sich mehr Sitzanteile der sozial-demokratischen 
Parteien mit einem schwachen Effekt $\left(b^{*}=0,04\right)$ positiv auf das Investitionsvolumen aus. Für die übrigen Größenklassen gilt mit stärkeren Effekten, dass bei mehr sozial-demokratischen Ratsm itgliedern das Investitionsvolum en geringer ist, als bei $\mathrm{m}$ ehr bürgerlichliberalen Sitzanteilen. Die Stärke der Part eieffekte (H4a) nimmt entgegen der Annahme nicht mit der Größenklasse ab. Diese Abweichung hinsichtlich der Parteidifferenzthese lässt zwei weitergehende Interpretationen zu: Erstens könnte politisches Agieren von Parteien auf kommunaler Ebene w eniger programmorientiert als von uns angenommen sein, wie es im Übrigen in Kommunalstudien häufig zu beobachten ist (vgl. H oltkamp 2008); zweitens könnte sich der Parteieinfluss auch dadurch verkehren, dass CDU und FDP durch Parteinetzw erke privilegierten Zugang zu Inform ationen aus der Landesregierung und den einschlägigen Ministerien hatten und dadurch im Mittelabruf bevorteilt waren. Beide Alternativerklärungen könnten aber nur durch eingehendere Fallstudien em pirisch geklärt werden und übersteigen den Rahmen der uns vorliegenden empirischen Evidenz.

Tabelle 6: Überprüfung der Hypothesen zum Investitionsvolumen

\begin{tabular}{|c|c|c|c|c|}
\hline & H1: Schulden & H2: Größe & H3: Verw.Kap. & $\begin{array}{l}\text { H4: soz.-dem. } \\
\text { Parteien }\end{array}$ \\
\hline GK1-3 & $3 /-/ x$ & $1 /+/ \sqrt{ }$ & $2 /-/ x$ & $4 /+/ \sqrt{ }$ \\
\hline GK 4 & $2 /-/ x$ & $1 /+/ \sqrt{ }$ & $4 /-/ x$ & $3 /-/ x$ \\
\hline GK 5 & $1 /+/ \sqrt{ }$ & $3 /+/ \sqrt{ }$ & $4 /-/ x$ & $2 /-/ x$ \\
\hline GK 6 & $2 I+N$ & $1 /+/ \sqrt{ }$ & $4 /+/ \sqrt{ }$ & $3 /-/ x$ \\
\hline GK 7 & $2 I+I N$ & $1 /+/ \sqrt{ }$ & $4 /+/ \sqrt{ }$ & $3 /-/ x$ \\
\hline $\begin{array}{l}\text { Durchschnittl. } \\
\text { Effektstärke }\end{array}$ & 2 & 1,4 & 3,6 & 3 \\
\hline
\end{tabular}

Anmerkungen: Die Stärke der Koeffizienten pro Größenklasse $\quad$ wird in dieser Tabelle durch die Zahlen 1-4 ausgedrückt, 1= stark 4= schwach; Die Effektricht ung wird durch + und - gekennzeichnet. Die Hypothesen lauteten: H1: Je besser ihre Haushaltslage, desto höher das Investitionsvolumen der Kommunen; H2: Je größer die Kommune, desto höher das Investitionsvolumen; H3: Je mehr Personal je Einwohner, desto höher das Investitionsvolumen; H4: Je mehr Stimmen die sozial-demokratisch en Parteien, desto höher das Investitionsvolumen; $\sqrt{ }=$ Hypothese bestätigt; $\mathbf{x}=$ Hypothese verworfen.

\subsection{Varianz der Mitnahmeeffekte}

Auch für die Analyse der Mitnahm eeffekte zeichnet sich in der bivariaten A nalyse (vgl. Tabelle 7) für die $456 \mathrm{~K}$ ommunen für die Mitnahm eeffekte angenom men w erden eine sehr unterschiedliche Ausprägung in der jewe iligen Höhe ab. Zur Erklärung der Varianz spielen auch hier die Größe und die Schulden die größte Rolle. Dabei ist die Wirkung der Haushaltslage umgekehrt als vermutet: Mit höherer Verschuldung gehen Mitnahmeeffekte einher. Bei einem Anstieg der G röße steigen die Mitnahm eeffekte an. Ebenso lassen sich bei Kommunen mit höheren Schulden auch höhere Mitnahmeeffekte feststellen. Darüber hinaus wirken sich die Verwaltungskapazität und ein höherer Sitzanteil sozial-demokratischer Parteien positiv auf die Höhe aus. 
Tabelle 7: Bivariater Zusammenhang: Mitnahmeeffekte als abhängige Variable

\begin{tabular}{|c|c|c|c|c|c|}
\hline Mitnahmeeffekte & Modell M1 & Modell M2 & Modell M3 & Modell M4 & Modell M5 \\
\hline \multicolumn{6}{|l|}{ Unabhängige Variablen } \\
\hline Größe & $\begin{array}{c}0,7381^{* * *} \\
(4,6448)\end{array}$ & & & & \\
\hline Schulden & & $\begin{array}{c}0,7652^{\star * *} \\
(0,0029)\end{array}$ & & & \\
\hline Verwaltungskapazität & & & $\begin{array}{c}0,3664^{\star * \star} \\
(3,40 \mathrm{e}+07)\end{array}$ & & \\
\hline Bürgerlich-liberale & & & & $-0,0564$ & \\
\hline Parteien & & & & $(522364,7)$ & \\
\hline $\begin{array}{r}\text { Sozial-demokratische } \\
\text { Parteien }\end{array}$ & & & & & $\begin{array}{c}0,3191^{* * *} \\
(961217,9)\end{array}$ \\
\hline $\mathrm{N}$ & 456 & 456 & 456 & 456 & 456 \\
\hline Adj. $R^{2}$ & 0,5438 & 0,5845 & 0,1324 & 0,0010 & 0,0999 \\
\hline
\end{tabular}

Anmerkungen: ${ }^{* *} \mathrm{p}<0,01,{ }^{* *} \mathrm{p}<0,05,{ }^{*} \mathrm{p}<0,1$. Angabe des Signifikanzniveau dient der Orientierung, jedoch nicht der Interpretation der Ergebnisse. Angegeben ist de $\mathrm{r}$ standardisierte Regressionskoeffizient, welcher die Effektrichtungen und -stärken angibt. Standardfehler in Klammern.

In der m ultiplen Regression in Modell M6, in der alle 456 Fälle m it Mitnahmeeffekten enthalten sind, bestätigen sich diese Erge bnisse: Der stärkste Erklärungsfaktor sind die Schulden $\left(b^{*}=0,4972\right)$. Von den Kommunen die Mitnahmeeffekte aufweisen, haben diejenigen Kommunen größere Mitnahm eeffekte, die höhere Schulden haben, als K ommunen mit niedrigeren Schulden. D es Weiteren haben größere Kommunen höhere Mitnahmeeffekte $\left(b^{*}=0,2645\right)$. Steigt die Verwaltungskapazität um eine Standardabweichung, so steigt die Höhe der Mitnahmeeffekte um 0,06 Standardabweichungen. Auch der Effekt des Sitzanteils von sozial-dem okratischen Parteien im kommunalen Entscheidungsorgan auf die Höhe der Mitnahmeeffekte ist sehr gering $\left(b^{*}=0,0491\right)$.

Analog zur Analyse der Höhe der Investitionsmittel wurde die Analy se getrennt nach Gemeindegrößenklassen wiederholt. Wie schon in Tabelle 3 gezeigt weisen dabei die Kommunen der Größe 50.000-100.000 Einwohner (GK4) den höchsten A nteil an K ommunen mit Mitnahmeeffekten auf.

Tabelle 8: Mitnahmeeffekte nach Gemeindegrößenklassen

\begin{tabular}{|c|c|c|c|c|c|c|}
\hline Mitnahmeeffekte & $\begin{array}{l}\text { Modell M6 } \\
\text { Alle }\end{array}$ & $\begin{array}{r}\text { Modell M7 } \\
\text { GK1-GK3 }\end{array}$ & $\begin{array}{l}\text { Modell M8 } \\
\text { GK4 }\end{array}$ & $\begin{array}{l}\text { Modell M9 } \\
\text { GK5 }\end{array}$ & $\begin{array}{l}\text { Modell M10 } \\
\text { GK6 }\end{array}$ & $\begin{array}{l}\text { Modell M11 } \\
\text { GK7 }\end{array}$ \\
\hline \multicolumn{7}{|l|}{ Unabhängige Variablen } \\
\hline Größe & $\begin{array}{c}0,2645^{\star * *} \\
(8,7982)\end{array}$ & $-2,732$ & $\begin{array}{c}0,8393 \\
(372,87)\end{array}$ & $\begin{array}{c}0,0597 \\
(117,18)\end{array}$ & $\begin{array}{l}0,0810 \\
(69,68)\end{array}$ & $\begin{array}{l}0,5097 \\
(17,12)\end{array}$ \\
\hline Schulden & $\begin{array}{c}0,4972^{* * *} \\
(0,0056)\end{array}$ & $-0,466769$ & $\begin{array}{l}-0,6064 \\
(0,1860)\end{array}$ & $\begin{array}{c}0,4771 \\
(0,0478)\end{array}$ & $\begin{array}{l}-0,2055 \\
(0,0248)\end{array}$ & $\begin{array}{l}-0,0269 \\
(0,0085)\end{array}$ \\
\hline Verwaltungskapazität & $\begin{array}{c}0,0647^{*} \\
(2,53 e+07)\end{array}$ & & $\begin{array}{c}-0,3220 \\
(6,60 \mathrm{e}+08)\end{array}$ & $\begin{array}{c}0,2731 \\
(2,25 \mathrm{e}+08)\end{array}$ & $\begin{array}{c}0,4812 \\
(7,79 \mathrm{e}+07)\end{array}$ & $\begin{array}{c}0,0572 \\
(9397692)\end{array}$ \\
\hline $\begin{array}{r}\text { Sozial-demokratische } \\
\text { Parteien }\end{array}$ & $\begin{array}{c}0,0491 \\
(691664,2)\end{array}$ & 3,27959 & $\begin{array}{c}0,0713 \\
(7,24 \mathrm{e}+07)\end{array}$ & $\begin{array}{c}-0,0855 \\
(1,08 \mathrm{e}+07)\end{array}$ & $\begin{array}{c}0,1029 \\
(1945384)\end{array}$ & $\begin{array}{c}-0,0575 \\
(266177,4)\end{array}$ \\
\hline $\bar{N}$ & 456 & 4 & 7 & 24 & 81 & 340 \\
\hline Adj. $R^{2}$ & 0,6117 & & & & & \\
\hline
\end{tabular}

Anmerkungen: Angegeben ist der standardisierte Regressionskoeffizient, sowie der Standardfehler. Modell M7 hat aufgrund der geringen Fallzahl stark verzerrte Beta-Koeffizienten und weist Multikollinearität auf. Für die Modelle M8- M11 w urde keine Multikollinearität festgeste llt. Auf die A ngabe des A dj.R² wird bei den Mes- 
sungen nach GK verzichtet, da mit zunehmender Fallzahl das Adj. $\mathrm{R}^{2}$ steigt und damit folglich kein verlässlicher Modellvergleich möglich ist.

In der genannten GK4 sind die mit Abstand stärksten Einflussfaktoren auf die Mitnahmeeffekte die $G$ röße $\left(b^{*}=0,8393\right)$ und die $H$ öhe der Schulden $\left(b^{*}=-0,6064\right)$ der $\mathrm{K}$ ommunen. Auch für die übrigen Größenklassen stellt die Größe einen Einflussfaktor dar, wobei sie in den GK5 und GK6 im Vergleich zu den anderen Prädiktoren nur den viertstärksten, für die G K7 jedoch w ieder den stärksten Effekt aufw eist. Eine defizitäre Haushaltslage stellt einen noch größeren negativen Einflussfaktor für alle Größenklassen der Kom munen dar. Ausnahme ist hier die fünfte G rößenklasse, in w elcher die Schulden einen stark positiven Effekt $\left(b^{*}=0,4771\right)$ auf die Höhe der Mitnahmeeffekte ausüben. Hypothese 7, welche besagt, dass $\mathrm{m}$ it zunehm ender V erwaltungskapazität die Mitnahm eeffekte ebenfalls zunehmen kann für die G K5, GK6 und G K7 bei vergleichsweise hohen Effektstärken bestätigt w erden. Für die größeren $K$ ommunen jedoch trifft die ursprüngliche A nnahme, dass $m$ it einer größeren Spezialisierung die Fähigkeiten und Kenntnisse steigen, das Antragsverfahren für die $\mathrm{G}$ emeinde vor teilhaft auszulegen, nicht zu. Tatsächlich scheint hier das Gegenargument der Spezialisierung, näm lich eine hohe Komplexität und Formalisierung des Verwaltungsverfahrens zu greifen.

Mehr sozial-demokratische Sitzanteile im kommunalen Rat w irken sich in G K4 und GK6 auf Mitnahmeeffekte fördernd aus. Fü r die GK5 und GK7 gilt, dass $m$ ehr bürgerlich-liberale Sitzanteile höhere Mitnahm eeffekte bedingen. Im Vergleich zu den anderen unabhängigen Variablen w eisen die Parteien die geringsten Effektstärken aus und sind somit schwächster Prädiktor für die Höhe der Mitnahmeeffekte. Wiederum ist ein Effekt der Gemeindegröße hinsichtlich der Effektstär ke nicht zu erkennen. Eine grafische Zusammenfassung der beschrieben Ergebnisse bezüglich der Mitnahm eeffekte als abhängige Variable zeigt Tabelle 9.

Tabelle 9: Überprüfung der Hypothesen zu den Mitnahmeeffekten

\begin{tabular}{lcccc} 
& H5: Schulden & H6: Größe & H7: Verw. Kap. & $\begin{array}{c}\text { H8: soz.-dem. } \\
\text { Parteien }\end{array}$ \\
\hline GK1-3 & $(-)$ & $(-)$ & $(+)$ & $4 /+/ \sqrt{ }$ \\
GK 4 & $2 /-/ \sqrt{ }$ & $1 /+/ \sqrt{ }$ & $3 /-/ \times$ & $3 /-/ \times$ \\
GK 5 & $1 /+/ x$ & $4 /+/ \sqrt{ }$ & $2 /+/ \sqrt{ }$ \\
GK 6 & $2 /-/ \sqrt{ }$ & $4 /+/ \sqrt{ }$ & $1 /+/ \sqrt{ }$ & $3 /+/ \sqrt{ }$ \\
GK 7 & $4 /-/ \sqrt{ }$ & $1 /+/ \sqrt{ }$ & $3 /+/ \sqrt{ }$ & $2 /-/ \times$ \\
Durchschnittliche & 2,25 & 2,5 & 2,25 & 3 \\
Effektstärke & & & \\
\hline
\end{tabular}

Anmerkungen: Die Stärke der Koeffizienten pro Größenklasse $\quad$ wird in dieser Tabelle durch die Zahlen 1-4 ausgedrückt, 1=stark 4=schwach; Die Effektrichtung wird durch + und - gekennzeichnet.

Die Hypothesen lauteten: H5: Je schwächer die Haushaltslage der Kommunen, desto unwahrscheinlicher sind die Mitnahmeeffekte; H6: Je größer die Kommune, desto wahrsc heinlicher sind die Mitnahmeeffekte; H7: Je mehr Personal je Einwohner, desto stärker sind die Mitnahmeeffekte; H8: Je mehr Stimmen die sozialdemokratischen Parteien, desto stärker die Mitnahmeeffekte; $\sqrt{ }=$ Hypothese bestätigt; $\mathrm{x}=$ Hypothese verworfen. 


\section{Schluss: Wer hat, dem wird manchmal (aber nur manchmal) gegeben!}

Ausgangspunkt dieses Beitrags w ar die Frage nach den Verteilungswirkungen und nichtintendierten Effekten distributiver Politiken am Beispiel der Im plementation des $\mathrm{Zu}-$ kunftsinvestitionsgesetzes in baden-württe mbergischen Kommunen. Ausgehend von theoretischen Hypothesen wurden zwei typische Probleme eingehend untersucht: Ungleichheiten in der Mittelverteilung und Mitnahmeeffekte.

Die Unterschiede im Investitionsvolum en der Kom munen lassen sich hauptsächlich durch die G emeindegröße, darüber hinaus aber auch über die Schulden und die Verwaltungskapazität erklären. Hinsichtlich des Ei nflusses der kom munalpolitischen Konstellationen sind die Ergebnisse nicht eindeutig und lassen $\mathrm{w}$ ie gesehen mehrere Interpretationen zu. Die in Baden-Württem berg durchgeführte distributive Verteilung der Mittel offenbart dabei aber nicht w ie ursprünglich angenommen extreme Verlierer- oder Gewinnerkommunen. Die Mittel wurden angesichts der Herausforderung einer kurzfristigen Umsetzung relativ effektiv und effizient - im Sinne eines schnellen Mittelabflusses - an die Kommunen verteilt. Dies heißt jedoch nicht, dass durch den gesetzten Anreiz die Programmziele vollständig erfüllt wurden. Der relativ hohe Anteil an Mitnahmeeffekten deutet darauf hin, dass Kom munen Mittel beantragt haben, um kurzfristig ihren Haushalt zu sanieren und nicht um in zukunftsorientierte Projekte zu investieren. D iese Kommunen haben die Program mziele durchaus strategisch an ihre Situation angepasst. Dabei waren grundsätzlich die Kommunen mit hoher Verwaltungskapazität und größerem finanziellen Spielraum im Vorteil, so dass unsere Ausgangsannahme der Triftigkeit des Matthäusprinzips zutrifft. Die Regierungspräsidien, die die Maßnahmen bewilligten, fungierten dabei als Erfüllungsgehilfen - was auch die auf den ersten Blick kontraintuitiven Effekte der parteipolitischen Konstellationen vor Ort erkl ären mag. Die Tatsache, dass einige Kom munen ihre Spielräum e ausgenutzt haben, deutet darauf hin, dass die eigentlichen Problemlagen der Kommunen - die desolate Haushaltslage - durch die distributive Politik des Kommunalen Investitionsprogramms zunächst nicht ausreichend berücksichtigt wurde.

Der Teil der Mittel, der nicht über Pauschal en vergeben wurde, wurde in einem Verfahren verteilt, das Kom munen $m$ it einer höheren Verwaltungskapazität Vorteile verschaffte. Ob derartige Mitnahmeeffekte von den „, Machern“ des Kom munalen Investitionsprogramms auf Bundes- und Landesebene bereits im Voraus beabsichtigt war muss hier offen bleiben. Gegen diese Annahme spricht jedoch die Abschaffung des $\$ 3 \mathrm{a}$ des ZuInvG. Erst im Frühjahr 2010 wurde das Zusätzlichkeitserfordernis des ZuInvG gelockert, sodass nur die Vorhaben, nicht aber generell die Investitionen zusätzlich sein $m$ ussten. Die erkannten Mitnahmeeffekte wurden damit sozusagen legalisiert, was zur Folge hatte, dass die von da an offiziellen Program mziele nicht so sehr verletzt w urden, wie sie zuvor in der ursprünglichen V ersion des ZuInvG verankert waren. Dies hatte auch den Vorteil, dass der Landes- und der Bundesrechnungshof weniger Fälle wegen Verstößen ahnden müssen, was bei der großen Zahl an Kommunen vermutlich sehr lange Zeit und hohe Kosten beansprucht hätte. D er G rund für die $\ddot{A}$ nderung des $\S 3$ a ZuInvG liegt sehr w ahrscheinlich aber auch darin, dass aus dem laufenden Prozess gelernt wurde: Es wurde erkannt, dass die Problem e der Kom munen nicht nur im Fehlen von Mitteln für Zukunftsinvestitionen lagen, sondern durch strukturelle Haushaltsdefizite generell gehindert wurden, 
in Infrastruktur zu investieren. Mit der Ände rung des Planzieles der zusätzlichen Investitionen wurde som it auf die Gegen-Im plementation eingegangen. Den Kom munen wurden Freiräume zugestanden, die ansonsten als Planzielverfehlungen hätten geahndet werden müssen.

Die Mitnahmeeffekte sind in vorliegendem Fall also, nicht wie in der Theorie angenommen grundsätzlich nicht-intendiert, sondern nach einem Feedbackloop, ab dem Zeitpunkt der Abschaffung des $\S 3$ a ZuInvG in die Program mumsetzung sozusagen integriert worden (vgl. Pressman/Wildavsky 1984, S. 232ff). Inwiefern aber das Ziel erreicht wurde, mit den Mitteln aus dem Kommunalen Investitionsprogramm tatsächlich zukunftsfähige Investitionen zu fördern, um den Kom munen nachhaltig aus der finanziellen Problemlage zu helfen, bleibt bei einer Veränderung der ursprünglichen Planziele fraglich. Für zukünftige Studien würde es sich anbieten, den Implementationsprozess weiterer Bundesländer zu analysieren, um Vergleichswerte und ein deutschlandweites Bild der Implementation des Zukunftsinvestitionsgesetztes so wie der dam it verbundenen steuerungspolitischen Varianten zu erhalten. D ie finanzielle Situation der K ommunen in Baden-Württemberg ist vergleichsweise gut, so dass sich in anderen Bundesländern noch stärkere Zielverschiebungen ergeben könnten.

\section{Literatur}

Barabas, György/Döhrn, Roland/Ge bhardt, Heinz/Schmidt, Torsten, 2009: Was bringt das Konjunkturpaket II?, in: Wirtschaftsdienst, 89, S. 128-132.

Barabas, György/Döhrn, Roland/Gebhardt, Heinz, 2011: Was brachte das Konjunkturpaket II?, in: Wirtschaftsdienst, 91, S. 496-498.

Behnke, Joachim, 2005: Lassen sich Signifika nztests auf Vollerhebungen anwenden? Einige essayistische Anmerkungen, in: Politische Vierteljahresschrift, 46, S. 1-15.

Gatzer, Werner, 2009: Gesetz zur Um setzung von Zukunfts investitionen der Kom munen und Länder. Brief an die Finanzminister de r Länder vom 23.03.2009, Berlin: B undesministerium der Finanzen. Online verfügbar unter: http://www.im.nrw.de/bue/doks/bmfzu104gg.pdf, Stand: 23.11.2010.

Bogumil, Jörg/Grohs, Stephan/Kuhlmann, Sabine/Ohm, Anna K. , 2007: Zehn Jahre Neues Steuerungsmodell. Eine Bilanz kommunaler Verwaltungsmodernisierung, Berlin: Edition Sigma.

Bogumil, Jörg/Grohs, Stephan/Reiter, Renate, 2008: Möglichkeiten und Grenzen nationaler Stadtpolitik - eine politikwissenschaftliche Perspektive, in: Raumforschung und Raumordnung, 66, S. 118-139.

Bogumil, Jörg/Jann, Werner, 2009: Verwaltung und Verwaltungswissenschaft in Deutschland. Einführung in die Verwaltungswissenschaft, Wiesbaden: VS Verlag.

Brenke, Karl/Dre ger, Christian/Kooths, Stefan/Kuzin, Vladimir/Weber, Sebastian/Zinsmeister, Florian, 2009: Grundlinien der Wirtschaft sentwicklung 2009/2010, Berlin: Deutsches Institut für Wirtschaftsforschung.

DeLeon, Peter/DeLeon, Linda, 2002: What Ever Happened to Polic y Implementation? An Alternative Approach, in: Journal of Public Administration Research and Theory, 12, S. 467-492.

Elmendorf, Douglas W./Furman, Jason, 2008: If, When, How: A Primer on Fiscal Stimulus, Washington: Brookings Institution.

Finanzministerium Baden-Württemberg, 2010a: Konjunkturprogram me - Investitionen und Verteilung der Mittel. Online verfügbar unter: http://www.fm.baden-wuerttemberg.de/de/Investitionsvolumen und_Verteilung_der_Mittel/203548.html, Stand: 23.11.2010.

Finanzministerium Baden-Württemberg, 2010b: Konjunkturprogramme - Häufig gestellte Fragen. Online verfügbar unter: http://www.fm.baden-wuerttemberg.de/fm7/2347/FAQ_Internet_03042009.pdf, Stand: 23.11.2010 
Finanzministerium Baden-Württemberg, 2010c: Konjunkturprogramme - Investitionsvolum en und Verteilung der Mittel. Zukunftsinvestitionsprogra mm: Aufteilung der Bundesm ittel auf Land und Kommunen. Online verfügbar unter: http://www.fm.b aden-wuerttemberg.de/fm7/2347/Uebersicht1 _Zukunftsinvestitionsprogramm_Auftteilung_der_Bundesmittel_auf_Land_und_Kommunen.pdf, Stand: 23.11.2010.

Finanzministerium Baden-Württemberg, 2010d: Konjunkturprogramme. Anmeldeverfahren für die Bildungsinfrastruktur- und Infrastrukturpauschale. Online verfügbar unter: http://www.fm.baden-wuerttemberg. de/fm7/2347/\%DCbersicht\%206 Anmeldeverfahren\%20 2 09032009.pdf"wuerttemberg.de/fm7/2347 /\% DCbersicht\%206_Anmeldeverfahren\%20_2_09032009.pdf, Stand: 23.11.2010.

Fuest, Winfried, 2009: Gute Schulden: Sind höhere Staatsschulden für das Konjunkturpaket gerechtfertigt?, in: Ifo Schnelldienst, 62, S. 3-8.

Gaul, Claus-Martin, 2008: Konjunkturprogramme in der Geschichte der Bundesrepublik Deutschland: Einordnung und Bewertung der Globalsteuerung von 1967 bis 1982, Wissenschaftliche Dienste des Deutschen Bundestages (WD 5 - 3010 - 009/09), Berlin: Deutscher Bundestag.

Grohs, Stephan, 2010: Modernisierung kom munaler Sozialpo litik. Anpassungsstrategien im Wohlfahrtskorporatismus, Wiesbaden: VS.

Hammerschmid, Gerhard/Stemmler, Robert, 2010: Programmsteuerung im Mehrebenensystem Deutschlands, in: Verwaltung \& Management, 3, S. 121-127.

Handrich, Lars/Meinen, Philipp/Pavel, Ferdinand/Wissmann, Daniel, 2009: Konjunkturpakete: Verpasste Chance für langfristiges Wachstum, in: Wochenbericht des DIW Berlin, 43, S. 748-752.

Hassel, Anke/Lütz, Susanne, 2010: Durch die Krise aus der Krise? Die neue Stärke des Staates, in: der moderne staat, 3, S. 251-274.

Heinelt, Hubert, 2009: Machen Besonderheiten von Policies einen Unterschied?, in: Schubert, Klaus/ Bandelow, Nils (Hrsg.), Lehrbuch der Politikfeldanalyse 2.0, München: Oldenburg, S. 115-130.

Heinze, Rolf G. , 2009: Rückkehr des Staates? Politische Ha ndlungsmöglichkeiten in unsicheren Zeiten, Wiesbaden: VS Verlag.

Hibbs, Douglas A. , 1977: Political Parties and Macroeconom ic Policy, in: American Political Science Review, 71, S. 1467-1487.

Hill, Michael/Hupe, Peter, 2009: Implementing Public Policy, London: Sage Publications Ltd.

Holtkamp, Lars, 2008: Kommunale Konkordanz- und Konkurrenzdemokratie - Parteien und Bürgermeister in der repräsentativen Demokratie, Wiesbaden: VS Verlag.

Holtkamp, Lars, 2010: Kommunale Haushaltspolitik bei leeren Kassen. Bestandsaufnahme, Konsolidierungsstrategien, Handlungsoptionen, Berlin: Edition Sigma.

Horn, Gustav/Hohlfeld, Peter/Tr uger, Achim/Zwiener, Rudolf, 2009: Höheres Tempo erforderlich - Zu den Wirkungen des Konjunkturpa kets II, Institut für Makroökonom ie und Konjunkturforschung, Düsseldorf: Hans Böckler Stiftung.

Klenk, Tanja/Nullmeier, Frank, 2010: Politische Krisentheorien und die Renaissance von Konjunkturprogrammen, in: der moderne staat, 3, S. 273-294.

Knill, Christoph/Tosun, Jale, 2010: Politikgestaltung in der Eur opäischen Union. Die Entstehung und Umsetzung der Dienstleistungsrichtlinie, Baden-Baden: Nomos.

Lowi, Theodore J., 1972: Four systems of policy, politics, and choice, in: Public Administration Review, 32, S. 298-310.

Mayntz, Renate, 1983: Die Im plementation politischer Progr amme: Theoretische Überlegungen zu einem neuen Forschungsgebiet, in: Mayntz, Renate (Hrsg.), Implementation politischer Programme II: Empirische Forschungsberichte, Königstein/Ts.: Anton Hain, S. 237-250.

Organisation für Wirtschaftliche Zu sammenarbeit und Entwicklung (OECD), 2010: Nach der Krise: Die Tragfähigkeit der öffentlichen Finanzen wiederhers tellen, in: OECD Wirtschaftsberichte: Deutschland 2010, OECD Publishing, S. 63-89.

Pavel, Ferdinand, 2009: Richtig Investieren I. Öffentliche Investitionen zur Erhöhung des langfristigen Wachstumspotentials in Deutschland, Berlin: DIWecon.

Pavel, Ferdinand/Proske, Sandra, 2009: Konjunkturprogramme: Investitionen ohne nachhaltige Wachstumswirkung, in: DIW-Wochenbericht 19/2009, S. 314-320. 
Pressman, Jeffrey L./Wildavsky, Aaron, 1984: Implementation, Berkeley, CA: University of California Press.

Rechnungshof Baden-Württemberg, 2011: Sonderbericht: Umsetzung des Zukunftsinvestitionsgesetzes: Bildungs- und Infrastrukturpauschalen, Stuttgart: Rechnungshof Baden-Württemberg.

Roos, Michael W. M., 2009: Die deutsche Fiskalpolitik während der Wirtschaftskrise 2008/2009, in: Perspektiven der Wirtschaftspolitik, 10, S. 389-412.

Schmidt, Sebastian/Prange, Florian/Schlegelmilch, Kai/Cottrell, Jacqueline/Görres, Anselm, 2009: Sind die deutschen Konjunkturpakete nachhaltig? Studie des Forum s Ökologisch- Soziale Marktwirtschaft im Auftrag des WWF, Berlin: Forum Ökologisch- Soziale Marktwirtschaft.

Wagschal, Uwe/Jäckel, T im, 2010: Öffentliche Finanzen im Stre sstest - Policy reaktionen auf die Finanz- und Wirtschaftskrise, in: der moderne staat, 3, S. 295-320.

Windhoff-Héritier, Adrienne, 1987: Policy-Analyse. Eine Einführung, Frankfurt am Main: Campus.

Wollmann, Hellmut, 1983: Implementation durch Gege nimplementation von unten, in: Mayntz, Renate (Hrsg.), Implementation politischer Programme II, Opladen: Westdeutscher Verlag, S. 168-196.

\section{Anschriften der Autorin und der Autoren:}

Jessika Schneider, MA, Universität Konsta nz, Fachbereich Politik- und Verwaltungswissenschaft, Fach D 91, D-78457 Konstanz

E-Mail: jessikaSchneider@gmx.de

Dr. Stephan Grohs, (Korrespondierender Autor)

Universität Konstanz, Fachbereich Politik- und Verwaltungswissenschaft, Fach D 91, D78457 Konstanz

E-Mail: stephan.grohs@uni-konstanz.de

Prof. Dr. Christoph Knill, Universität Kons tanz, Fachbereich Politik- und Verwaltungswissenschaft, Fach D 91, D-78457 Konstanz

E-Mail: christoph.knill@uni-konstanz.de) 